\title{
Detection of a Yersinia pestis gene homologue in rodent samples
}

Timothy A Giles, Alex D Greenwood, Kyriakos Tsangaras, Paul A Barrow, Duncan Hannant, Abu-Bakr Abu-Median, Lisa Yon

A homologue to a widely used genetic marker,pla,forYersinia pestishas been identified in tissue samples of two species of rat (Rattus rattusandRattus norvegicus) and of mice (Mus musculusandApodemus sylvaticus) using a microarray based platform to screen for zoonotic pathogens of interest. Samples were from urban locations in the UK (Liverpool) and Canada (Vancouver). The results indicate the presence of an unknown bacterium that shares a homologue for the pla gene of Yersinia pestis, so caution should be taken when using this gene as a diagnostic marker. 
1 Detection of a Yersinia pestis gene homologue in rodent samples

2 Timothy Andrew Giles (corresponding author) BSc MSc PhD

3 School of Veterinary Medicine and Science, University of Nottingham, Loughborough,

4 Leicestershire, United Kingdom.

5 Phone number: 01159516469 Email: timothy.giles@nottingham.ac.uk

6 Alex D Greenwood

7 Department of Wildlife Diseases, Leibniz Institute for Zoo and Wildlife Research, Berlin,

8 Germany, Department of Veterinary Medicine, Freie Universität Berlin, Berlin, Germany

9 Kyriakos Tsangaras

10 Department of Wildlife Diseases, Leibniz Institute for Zoo and Wildlife Research, Berlin,

11 Germany, Department of Translational Genetics, Cyprus Institute of Neurology and Genetics,

12 Nicosia, Cyprus

13 Paul A Barrow

14 School of Veterinary Medicine and Science, University of Nottingham, Loughborough,

15 Leicestershire, United Kingdom.

16 Duncan Hannant

17 School of Veterinary Medicine and Science, University of Nottingham, Loughborough,

18 Leicestershire, United Kingdom.

19 Abu-Bakr Abu-Median

20 School of Veterinary Medicine and Science, University of Nottingham, Loughborough,

21 Leicestershire, United Kingdom.

22 Lisa Yon 
23 School of Veterinary Medicine and Science, University of Nottingham, Loughborough,

24 Leicestershire, United Kingdom.

25

26 Abstract

27 A homologue to a widely used genetic marker, pla, for Yersinia pestis has been identified in

28 tissue samples of two species of rat (Rattus rattus and Rattus norvegicus) and of mice (Mus

29 musculus and Apodemus sylvaticus) using a microarray based platform to screen for zoonotic

30 pathogens of interest. Samples were from urban locations in the UK (Liverpool) and Canada

31 (Vancouver). The results indicate the presence of an unknown bacterium that shares a

32 homologue for the pla gene of Yersinia pestis so caution should be taken when using this gene as

33 a diagnostic marker.

34

35 Introduction

36 Yersinia pestis is the causative agent of plague in humans and, in the absence of antimicrobial

37 therapy, the mortality rate can approach 100\%. In large parts of the world the threat from $Y$.

38 pestis has declined substantially over time as a result of improvements in living conditions and in

39 public health, including improved rodent control and antibiotics. However, a plague outbreak

40 following the release of a biological weapon is a potential risk. The presence of $Y$. pestis in small

41 rodent populations in which it is endemic (Ziwa et al., 2013, Eppinger et al., 2009, Biggins and

42 Kosoy, 2001) can cause human fatalities as a result of zoonotic transmission (ProMED-mail,

43 2014).

44 The Black rat (Rattus rattus) has been a major host of $Y$. pestis for centuries and can be a

45 reservoir for numerous other pathogens. Although most mammalian species can be infected 
46 experimentally with $Y$. pestis, many species fail to develop the high bacteraemia that is necessary

47 to infect the flea vectors. The majority of mammalian species are therefore likely to be dead end

48 hosts (Eisen and Gage, 2009).

49 Molecular methods, and in particular PCR, have been widely used to identify Y. pestis in

50 tissue samples and the plasminogen activator/coagulase (pla) gene, located on the pPCP1

51 plasmid has been used as a target in many studies (Loïez et al., 2003, Raoult et al., 2000, Zhang

52 et al., 2013, Ziwa et al., 2013). The pla gene is commonly used because it has a high copy

53 number in $Y$. pestis (186 per bacterium) and can be detected relatively easily (Parkhill et al.,

54 2001). The PcP plasmid, and the pla gene in particular, is involved in transmission of $Y$. pestis

55 (Broekhuijsen et al., 2003). The protein encoded by the pla gene induces fibrinolysis and

56 degrades the extracellular matrix and basement membranes, these activities are thought to disrupt

57 the host's ability to contain the bacteria (Sebbane et al., 2006). The acquisition by Y. pestis of

$58 \mathrm{PcP}$, and of another virulence plasmid, the pMT, is thought to have contributed to the

59 evolutionary transformation of $Y$. pestis from the mainly gut-associated Yersinia enterocolitica,

60 into a highly host-adapted mammalian blood-borne pathogen (Eppinger et al., 2010).

61 Results and Discussion

62 Probes specific to $Y$. pestis hybridised with samples from a subset of each of the rodent

63 species tested (12/33 R. rattus, 48/834 R. norvegicus, 3/163 A. sylvaticus, 2/35 M. musculus)

64 giving a total of 65/1065 samples (6.1\%) which tested positive on the array. However, none of

65 the generic Yersinia probes hybridised in those samples for which a positive signal was recorded

66 for the $Y$. pestis specific probes.

67 Further testing was then carried out at the University of Nottingham, including real-time PCR

68 which targeted another region of the $Y$. pestis genome, the cafl gene, for which primers used 
were identified from the literature (Janse et al., 2010). A subset (23 samples) of the arraypositive samples was tested further with primers for pla and cafl. Of these samples, 12 were positive for the pla gene and all were negative for the cafl gene. A total of 30 array-positive samples were also sent to colleagues in Berlin for further analysis by in solution-based sequence hybridisation, as described previously (Tsangaras et al., 2014). Briefly a DNA extract from the samples was fragmented and an aliquot was used to produce illumina libraries following a custom protocol (Meyer and Kircher, 2010). PCR amplicons from Y. pestis genes were used to enrich specific target DNA sequences in the rodent samples, the genes and primers used to make the baits are shown in Table 3. The enriched samples were then sequenced using an illumina Miseq. The results indicated that the majority of reads aligned with the rat genome, and only a small number of reads aligned with the pla gene. No other reads mapped to any other gene from Y. pestis.

Although a homologue to the pla gene has previously been reported in bacteria found in $R$. rattus and $R$. norvegicus from the Netherlands (Janse et al., 2013), this is the first time, to the author's knowledge, that the bacterial homologue has been reported in M. musculus and A. sylvaticus. The potential discovery of a pla gene homologue in other rodent species, and on another continent than the species and locations in which it has previously been reported, suggests that the homologue could be more widely distributed than previously thought and may cause difficulties in accurate $Y$. pestis detection. The results found here supports other work which suggests that markers other than the pla gene should be included to help avoid false positive results when screening for $Y$. pestis, as has been stressed by Janse et.al (Janse et al., 2013). This was recently confirmed by Hänsch et. al, as they found evidence that the pla gene is present in some strains of Escherichia coli and Citrobacter koseri (Hänsch et al., 2015). It is not 
92 clear why the homologue was present in a larger percentage of $R$. rattus samples than in the other

93 species tested; perhaps $R$. rattus carries more E. coli or C. koseri, but this is something that needs

94 to be investigated further.

95 Materials and Methods

96 This work was part of a EU project (FP7 WildTech) to develop and use a microarray to detect

97 zoonotic pathogens in rodent tissues. A sequence of the pPCP1 plasmid of the $Y$. pestis genome

98 (CP000310.1) was obtained from the NCBI database for microarray probe design. Probes were

99 designed using two publicly available software packages: OligoWiz

100 (http://www.cbs.dtu.dk/services/OligoWiz/) and Unique Probe Selector

101 (http://array.iis.sinica.edu.tw/ups/). All probes were checked for suitability using an in silico

102 BLAST analysis. The results of the in silico analysis at the time indicated that the probe

103 sequences were specific to $Y$. pestis and no cross-hybridisation should occur with eukaryotic or

104 prokaryotic species. Primers were designed using the software Primer3

105 (http://bioinfo.ut.ee/primer3-0.4.0/). The sequence of each oligonucleotide probe specific to $Y$.

106 pestis is given in Table 1. During the confirmatory testing, both real-time PCR and end-point

107 PCR were used. The primers used in standard end-point PCR and real-time PCR are shown in

108 Table 2. These probes were evaluated thoroughly for specificity using reference samples of

109 genomic DNA from Y. pestis NCTC5923 Type strain and non-related pathogens before

110 screening took place. The microarray platform used was the ArrayStrip from Alere Technologies

111 GmbH (Jena, Germany).

112 Four different rodent species (R. rattus, R. norvegicus, Mus musculus and Apodemus

113 sylvaticus) were screened for a number of zoonotic pathogens. Tissue samples were obtained

114 from Vancouver (Canada), Liverpool (UK), and Lyon (France) as part of other studies. 
115 Automated nucleic acid extraction was performed on the samples using the QIAcube (Qiagen,

116 Hilden, Germany) and the kit (Cador Pathogen Mini Kit (Qiagen)). Liver, kidney and lung

117 tissues were available from each rodent sampled from Vancouver and Lyon, and extracted

118 nucleic acid from each tissue was pooled to make a single sample per individual animal which

119 was tested on the array. Only liver and kidney samples were available from the rodents sampled

120 from Liverpool, and again, extracted nucleic acid was pooled to make a single sample. Figure 1

121 depicts the sequence enrichment and microarray hybridisation used in this study.

125 Key findings

126 1. Sequences homologous to the pla gene, which is present in $Y$. pestis, have been found in 127 samples from several rodent species, in the absence of $Y$. pestis.

128 2. PCR, microarray and sequencing data suggest that these sequences may be present in 129 environmental bacteria.

130 3. Caution is warranted in interpreting screening results for detection of Y. pestis when the pla 131 gene is used as the sole marker for the presence of the pathogen.

133 Acknowledgements

134 We would like to thank Chelsea Himsworth, Florence Ayral, and Kieran Pounder for providing 135 the tissue samples. The authors would also like to thank Brendan Wren from the London School 136 of Hygiene and Tropical Medicine for providing the $Y$. pestis DNA sample. Finally, the authors 
137

138

139

140

141

142

143

144

145

146

147

148

149

150

151

152

153

154

155

156

157

158

159

160

161

162

163

164

165

166

167

168

169

170

171

172

173

174

175

176

177

178

179

would like to thank Tom Giles from the University of Nottingham's Advanced Data Analysis

Centre.

\section{References}

BIGGINS, D. E. \& KOSOY, M. Y. 2001. Influences of introduced plague on North American mammals: implications from ecology of plague in Asia. Journal of Mammalogy, 82, 906-916.

BROEKHUIJSEN, M., LARSSON, P., JOHANSSON, A., BYSTRÖM, M., ERIKSSON, U., LARSSON, E., PRIOR, R. G., SJÖSTEDT, A., TITBALL, F. W. \& FORSMAN, M. 2003. Genome-wide DNA microarray analysis of Francisella tularensis strains demonstrates extensive genetic conservation within the species but identifies regions that are unique to the highly virulent $F$. tularensis subsp. tularensis. Journal of Clinical Microbiology, 41, 2924-2931.

EISEN, R. J. \& GAGE, K. L. 2009. Adaptive strategies of Yersinia pestis to persist during inter-epizootic and epizootic periods. Veterinary Research, 40.

EPPINGER, M., GUO, Z., SEBASTIAN, Y., SONG, Y., LINDLER, L. E., YANG, R. \& RAVEL, J. 2009. Draft Genome Sequences of Yersinia pestis Isolates from Natural Foci of Endemic Plague in China. Journal of Bacteriology, 191, 7628-7629.

EPPINGER, M., WORSHAM, P. L., NIKOLICH, M. P., RILEY, D. R., SEBASTIAN, Y., MOU, S., ACHTMAN, M., LINDLER, L. E. \& RAVEL, J. 2010. Genome Sequence of the Deep-Rooted Yersinia pestis Strain Angola Reveals New Insights into te Evolution and Pangenome of the Plague Bacterium. Journal of Bacteriology, 192, 1685-1699.

HÄNSCH, S., CILLI, E., CATALANO, G., GRUPPIONI, G., BIANUCCI, R., STENSETH, N. C., BRAMANTI, B. \& PALLEN, M. J. 2015. The pla gene, encoding plasminogen activator, is not specific to Yersinia pestis. $B M C$ research notes, 8, 535.

HINNEBUSCH, B. J., FISCHER, E. R. \& SCHWAN, T. G. 1998. Evaluation of the Role of the Yersinia pestis Plasminogen Activator and Other Plasmid-Encoded Factors in Temperature-Dependent Blockage of the Flea. Journal of Infectious Diseases, 178, 1406-1415.

JANSE, I., HAMIDJAJA, R. A., BOK, J. M. \& VAN ROTTERDAM, B. J. 2010. Reliable detection of Bacillus anthracis, Francisella tularensis and Yersinia pestis by using multiplex qPCR including internal controls for nucleic acid extraction and amplification. BMC Microbiology, 10.

JANSE, I., HAMIDJAJA, R. A. \& REUSKEN, C. 2013. Yersinia pestis Plasminogen Activator Gene Homolog in Rat Tissues. Emerging Infectious Diseases, 19, 342-344.

LOÏEZ, C., HERWEGH, S., WALLET, F., ARMAND, S., GUINET, F. \& COURCOL, R. J. 2003. Detection of Yersinia pestis in Sputum by Real-Time PCR. Journal of Clinical Microbiology, 41, 4873-4875.

MEYER, M. \& KIRCHER, M. 2010. Illumia Sequencing Library Preparation for Highly Multiplexed Target Capture and Sequencing. Cold Spring Harbor Protocols, pdb.prot5448.

NORKINA, O. V., KULICHENKO, A. N., GINTSBURG, A. L., TUCHKOV, I. V., POPOV, Y. A., AKSENOV, M. U. \& DROSDOV, I. G. 1994. Development of a diagnostic test for Yersinia pestis by the polymerase chain reaction. Journal of Applied Microbiology, 76, 240-245.

PARKHILL, J., WREN, B. W., THOMSON, N. R., TITBALL, R. W., HOLDEN, M. T. G., PRENTICE, M. B., SEBAIHIA, M., JAMES, K. D., CHURCHER, C., MUNGALL, K. L., BAKER, S., BASHAM, D., BENTLEY, S. D., BROOKS, K., CERDENO-TARRAGA, A. M., CHILLINGWORTH, T., CRONIN, A., DAVIES, R. M., DAVIS, P., DOUGAN, G., FELTWELL, T., HAMLIN, N., HOLROYD, S., JAGELS, K., KARLYSHEV, A. V., LEATHER, S., MOULE, S., OYSTON, P. C. F., QUAIL, M., RUTHERFORD, K., SIMMONDS, M., 
SKELTON, J., STEVENS, K., WHITEHEAD, S. \& BARRELL, B. G. 2001. Genome sequence of Yersinia pestis, the causative agent of plague. Nature, 413, 523-527.

PROMED-MAIL 2014. Plague-China (02): (Gansu), Fatality. Pro-MED-mail 27.

RAOULT, D., ABOUDHARAM, G., CRUBÉZY, E., LARROUY, G., LUDES, B. \& DRANCOURT, M. 2000. Molecular identification by "suicide PCR" of Yersinia pestis as the agent of Medieval Black Death. PNAS, 97, 12800-12803.

RIEHM, J. M., RAHALISON, L., SCHOLZ, H. C., THOMA, B., PFEFFER, M., RAZANAKOTO, L. M., DAHOUK, S. A., NEUBAUER, H. \& TOMASO, H. 2011. Detection of Yersinia pestis using real-time PCR in patients with suspected bubonic plague. Molecular and Cellular Probes, 25, 8-12.

SEBBANE, F., JARRETT, C. O., GARDNER, D., LONG, D. \& HINNEBUSCH, B. J. 2006. Role of the Yersinia pestis plasminogen activator in the incidence of distinct septicemic and bubonic forms of fleaborne plague. PNAS, 103, 5526-5530.

TSANGARAS, K., SIRACUSA, M. C., NIKOLAIDIS, N., ISHIDA, Y., CUI, P., VIELGRADER, H., HELGEN, K. M., ROCA, A. L. \& GREENWOOD, A. D. 2014. Hybridisation Capture Reveals Evolution and Conservation across the Entire Koala Retrovirus Genome. PloS One, 9, e95633.

ZHANG, Z., LIANG, Y., YU, D., XIA, L. \& HAI, R. 2013. Development of a multiplex polymerase chain reaction (PCR) with an internal control method to detect Yersinia pestis in the plague foci surveillance. African Journal of Microbiology Research, 7, 698-700.

ZIWA, M. H., MATEE, M. I., KILONZO, B. S. \& B.M., H. O. 2013. Evidence of Yersinia pestis DNA in rodents in plague outbreak foci in Mbulu and Karatu Districts, northern Tanzania. African Journals Online, 15. 
201 Table 1. Y. pestis specific oligonucleotide probes used on the Alere ArrayStrip

\begin{tabular}{|c|c|c|c|c|}
\hline Probe & Sequence (5'-3') & Pathogen & Gene target & Position* \\
\hline Y.pestis_Owiz_117 & TACAGATCATATCTCTCTTTTCATCCTCCCCTAGCGGGGAGGATGTCTGTGGAAAGGAGG & Y.pestis & pPCP1 & $8781-8840$ \\
\hline Y.pestis_Owiz_120 & TGTTGTCCGCTAGGACGATGCGATTTCGGTTATTATTCAGAATGTCTTCGTTCTCTTTC & Y.pestis & pPCP1 & $6626-6684$ \\
\hline Y.pestis_Owiz_121 & TGTCCGGGAGTGCTAATGCAGCATCATCTCAGTTAATACCAAATATATCCCCTGACAGC & Y.pestis & pPCP1 & $7878-7936$ \\
\hline Y.pestis_Owiz_127 & GTGGAGATTCTGTCTCTATTGGCGGAGATGCTGCCGGTATTTCCAATAAAAATTATACTG & Y.pestis & pPCP1 & $8688-8747$ \\
\hline Y.pestis_Owiz_129 & GAATCGCGCCCGGATATGTTTTAACGCGATTTTCAGACTCAGACAAATTCAGCAGAAT & Y.pestis & pPCP1 & $9990-10047$ \\
\hline Y.pestis_Owiz_147 & TCGCTGGCTAAAAAGTACCATCCACATGCTCAACCCTATAACCTGTAGCTTACCCCAC & Y.pestis & pPCP1 & $9583-9640$ \\
\hline YpestisUPS_785 & AATAGGTTATAACCAGCGCTTTTCTATGCCATATATTGGACTTGCAGGCCAGTATCGCAT & Y.pestis & pPCP1 & $8392-8451$ \\
\hline YpestisUPS_786 & AATGATGAGCACTATATGAGAGATCTTACTTTCCGTGAGAAGACATCCGGCTCACGTTAT & Y.pestis & pPCP1 & $8510-8569$ \\
\hline YpestisUPS_787 & TAAATTCAGCGACTGGGTTCGGGCACATGATAATGATGAGCACTATATGAGAGATCTTAC & Y.pestis & pPCP1 & $8479-8538$ \\
\hline Y.pestisUPS_788 & AGCCCGACCACTGCGCCTTATCCGGTAACTATCGTCTTGAGTCCAACCCGGTAAGACACG & Y.pestis & pPCP1 & $4977-5036$ \\
\hline YpestisUPS_789 & TCATCCTCCCCTAGCGGGGAGGATGTCTGTGGAAAGGAGGTTGGTGTTTGACCAACCTTC & Y.pestis & $\mathrm{pPCP}$ & $8801-8860$ \\
\hline YpestisUPS_790 & AAAGGACAGCATTTGGTATCTGTGCTCCACTTAAGCCAGCTACCACAGGTTAGAAAGCCT & Y.pestis & $\mathrm{pPCP}$ & $5129-5188$ \\
\hline YpestisUPS_791 & AAGGAGTGCGGGTAATAGGTTATAACCAGCGCTTTTCTATGCCATATATTGGACTTGCAG & Y.pestis & $\mathrm{pPCP}$ & $8379-8438$ \\
\hline YpestisUPS_792 & TTTGTACCGAGAACCTTTCACGGTATCGGCATATGGCCTGGGTAACTCAGGTCCGTAAAC & Y.pestis & $\mathrm{pPCP}$ & $9451-9510$ \\
\hline
\end{tabular}

202 *The nucleotide position of each probe is based on the CP000310.1 Yersinia pestis Antiqua plasmid pPCP

203 Table 2. $Y$. pestis specific primers for standard end-point PCR and real-time PCR 


\begin{tabular}{lllll}
\hline Sequence (5'-3') & Reverse Primer & Sequence (5'-3') & Probe & Sequence (5'-3') \\
\hline CCCGAAAGGAGTGCGGGTAA & Y.pes/pPCP/8902/R & CGCCCCGTCATTATGGTGAA & N/A & N/A \\
\hline CCAGCCCGCATCACT & cafpri_r & ATCTGTAAAGTTAACAGATGTGCTAGT & Tqpro_caf & JOE- \\
AGCGTACCAACAAGTAATTCTGTATCGATC & BHQ1 \\
\hline AGACATCCGGCTCACGTTAT & Y.pes_pPCP_R & GAGTACCTCCTTTGCCCTCA & Y.pes_pPCP_Pr FAM-CACCTAATGCCAAAGTCTTTGCGGA- & TAMRA
\end{tabular}

204 ** The nucleotide position of the Y.pes_pPCP_F and Y.pes_pPCP_R primers based on the CP000310.1 Yersinia pestis Antiqua 205 plasmid pPCP

$206 * * *$ The nucleotide position of the cafpri_f and cafpri_r primers based on the KF682424.1 Yersinia pestis strain S1 plasmid pMT1

207 capsule protein F1 (cafl) gene 
208 Table 3. $Y$. pestis primers used to prepare baits for Illumina Miseq sequencing.

209

210

Primer name

Primer Sequence

Target Reference

211 F1

CAGTTCCGTTATCGCCATTGC

caf1 (Norkina et

212 F2

TATTGGTTAGATACGGTTACGGT

al., 1994)

213 Ypfur1

GAAGTGTTGCAAAATCCTGCG

fur

(Hinnebusch

214 Ypfur2

AGTGACCGTATAAATACAGGC

et al., 1998)

215

216

YPtoxU

AGGACCTAATATGGAGCATGAC

Ymt

(Riehm et

217 YPtoxUR

CGTGATTACCAGGTGCAACA

al., 2011)

218

219

220

221

222

223

224 
A

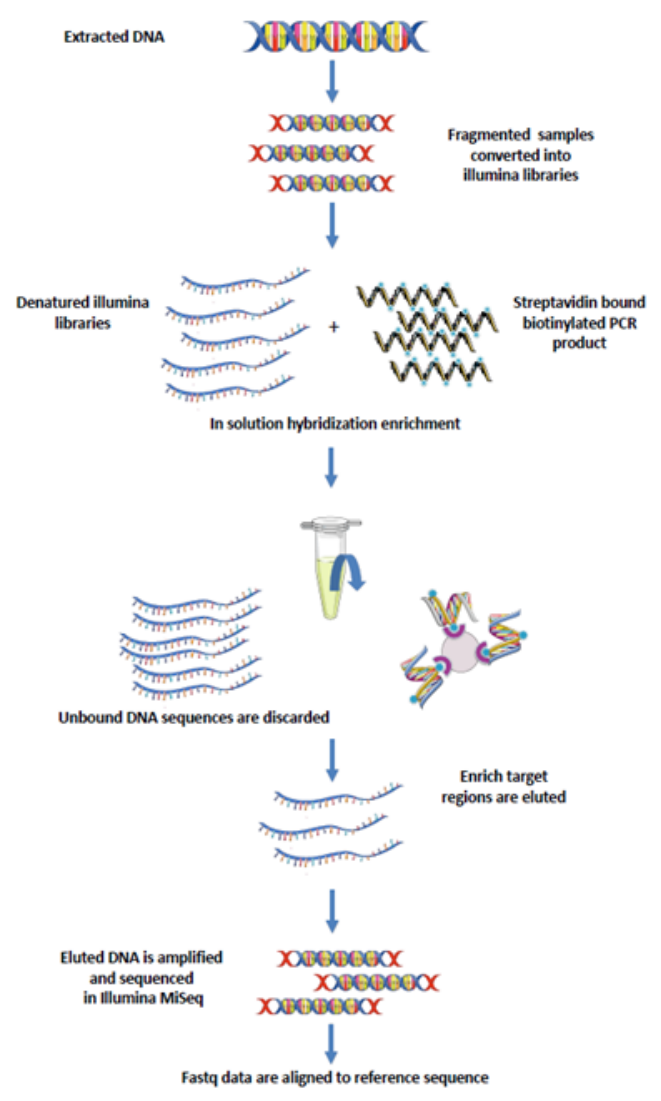

B

Extracted DNA $\quad$ XDOXDOOK

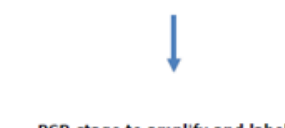

PCR stage to amplify and label

$\downarrow$

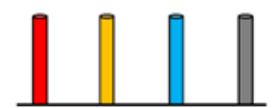

A microarray has unique probes
spotted onto its surface, these are fixed and immobile
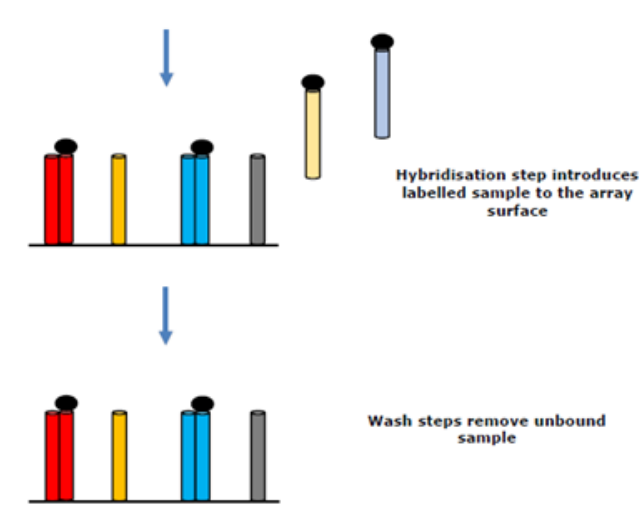

Wash steps remove unbound
sample

Figure 1. Sequence showing how extracted DNA is used for sequence enrichment capture (A) and microarray hybridisation (B). 
